\title{
Subvocal rehearsal of neutral and affective words interferes with left-hemisphere performance and facilitates right-hemisphere performance
}

\author{
GEORGE J. DEMAKIS and DAVID W. HARRISON \\ Virginia Polytechnic Institute and State University, Blacksburg, Virginia
}

\begin{abstract}
Kinsbourne's selective activation model was evaluated in a sample of 31 strongly right-dominant males. Priming manipulations were hypothesized to preferentially activate a hemisphere and to improve performance on an affect-recognition task in the contralateral visual field. Subvocal rehearsal of neutral words was the left-hemisphere manipulation, while subvocal rehearsal of affective words was the right-hemisphere task. Baseline performance was evaluated via a control condition. Relative to the control condition, both manipulations interfered with performance of the right visual field and facilitated that of the left visual field. These findings implicate an overloading of left-hemisphere processing capacity, but a priming of the right hemisphere. Left-hemisphere representation of both affective and neutral words and the dynamic balance of activation between hemispheres is proposed to account for these findings. Furthermore, a right-hemisphere advantage was observed for happy faces but not for angry faces, across all conditions. Perceptual asymmetries are proposed to account for this finding, though interpretative caution is required due to its observation in a dual-task paradigm.
\end{abstract}

Kinsbourne's selective activation model proposes that the hemispheres are asymmetrically activated or primedthat is, among right-handers, the left hemisphere is primed for verbal processing and the right hemisphere for visuospatial processing (Kinsbourne, 1980; Kinsbourne \& Byrd, 1985). During priming, the balance of activation between the hemispheres is altered and performance is hypothesized to improve for material presented to the primed hemisphere. For example, Kinsbourne (1970) found that subvocal verbal rehearsal facilitated gap detection in the right visual field (RVF), whereas across visual fields without a verbal load performance was equal. Van Strien and Bouma (1990) recently substantiated these findings and demonstrated the efficacy of verbal material in facilitating performance in the RVF.

Replication of these findings, however, has proved difficult. Boles (1979) found that subvocalization of six words interfered with RVF form recognition, but no interference was noted during two-word rehearsal. Gardner and Branski (1976) obtained a comparable pattern of results with six-word (Experiment 1) and three-word (Experiment 2) rehearsal. These and similar findings (see Moscovitch, 1986) suggest that priming or interference effects are largely a function of a manipulation's load or effort requirements (Kinsbourne \& Byrd, 1985). Under light loads, the secondary task (i.e., subvocalization) spreads activation, biases attention, and facilitates processing in the contralateral visual field. More demand-

The authors wish to thank two anonymous reviewers for their helpful comments. Reprint requests should be addressed to D. W. Harrison, Department of Psychology, Virginia Polytechnic Institute and State University, Blacksburg, VA 24061-0436. ing tasks overload a hemisphere and decrease its processing capacity for other tasks, particularly those that compete for similar cerebral areas (Demakis, Harrison, \& Campen, 1993; Kinsbourne \& Hisock, 1983). A number of experiments provide empirical support for this distinction (Bouma, 1987; Hellige \& Cox, 1976; Hellige, Cox, \& Litvac, 1979; Kinsbourne \& Byrd, 1985). Kinsbourne and Byrd (1985) required subjects to subvocally rehearse either no words, two words, four words, or six words during a visual recognition task. A significant quadratic trend for recognition accuracy was found in the RVF (i.e., recognition was poorer for no-word or six-word rehearsal, but was significantly better during two- or four-word rehearsal). Analogously, Hellige and Cox (1976) and Hellige et al. (1979) found that RVF priming and interference for shape identification varied with rehearsal load, while Bouma (1987) obtained similar effects for letter recognition. In all, RVF performance has been demonstrated to vary as a function of the load or effort requirements of verbal manipulations.

A smaller body of research has examined righthemisphere primes (see Moscovitch, 1986). Imagery has been demonstrated to effectively prime the right hemisphere and to facilitate left-visual-field (LVF) performance (Metzger \& Antes, 1976; Seamon \& Gazzaniga, 1973; cf. Van Strien \& Bouma, 1990). More importantly, affective material has also been employed as a right-hemisphere prime [see Borod (1992) for a review of right-hemisphere affective processing]. Brody, Goodman, Holm, Krinzman, \& Sebrechts (1987) used positive and negative affective words and faces as primes and found significant reaction-time (Experiments 1 and 2) and accuracy (Experiments 3 and 4) improvements for 
affective target stimuli presented in the LVF. Stimuli of both positive and negative valence primed the right hemisphere, but interfered with left-hemisphere performance. Bryden and Ley (1983) required subjects to memorize affective words between either of two laterality tasks - tachistoscopically presented faces or dichotic word listening. The subjects were instructed to "hold" the words in their memory while they were retested on the laterality task. Relative to the first presentation of the stimuli, significant shifts in favor of the right hemisphere were obtained in the second presentation. More recently, Van Strien and Morpurgo (1992) required subjects to subvocally rehearse threatening (emotional) or nonthreatening (neutral) words during a letter-recognition task. Compared with a control condition, emotional-word rehearsal improved accuracy in the LVF, whereas neutral words did so in the RVF. In brief, these dual-task experiments suggest that affective words may preferentially activate the right hemisphere and improve LVF performance.

The impact of affective words on right-hemisphere performance is inconsistent with Kinsbourne's proposal that verbal material selectively primes the left hemisphere. In fact, it might be argued that these stimuli actually induce negative affect, which is associated with right-hemisphere activation (Davidson, Ekman, Saron, Senulis, \& Friesen, 1990; see also Davidson, 1992). However, Bryden and Ley (1983), the only investigators to address this issue, found no affective alterations following the priming manipulations. Moreover, because wordrehearsal manipulations are of a limited duration and alternate frequently, it is difficult to submit that an affective state is actually induced. The above experiments suggest, rather, that affective verbal material may be right lateralized, independent of mood or experienced affect.

This position is supported by clinical research on aphasics. The ability to communicate via affective words may be retained following destruction of left-lateralized propositional speech centers (Gainotti, 1972). Emotionally loaded lexical materials have also been found to facilitate aphasics' performance on auditory comprehension (Boller, Cole, Vrtunski, Patterson, \& Kim, 1979) and oral reading and writing tasks (Landis, Graves, \& Goodglass, 1982). Additionally, impaired performance by right-hemisphere-damaged patients has been observed on a variety of emotionally based lexical tasks, including identification and discrimination of emotional words and sentences (Borod, Andelman, Obler, Tweedy, \& Welkowitz, 1992). This clinically based evidence thus also implicates the potential right lateralization of affective verbal material.

The following experiment was based on the selective activation model and utilized concurrent processing tasks to selectively prime each hemisphere. The two priming tasks - namely, subvocal rehearsal of either neutral or affective words - were hypothesized to alter functional cerebral asymmetries in a paradigm sensitive to hemispheric processing differences (Harrison \&
Gorelczenko, 1990). The paradigm required subjects to identify the affect-happy or angry-of tachistoscopically presented faces. The two hypotheses tested were (1) that, relative to the control condition, rehearsal of neutral words would prime the left hemisphere and improve RVF performance, and (2) that, relative to the control condition, rehearsal of affective words would prime the right hemisphere and improve LVF performance.

\section{METHOD}

\section{Subjects}

Thirty-one strongly right-dominant males (mean age $=19.26$ years, $S D=1.03$, range $=18-22$ ) from the undergraduate subject pool participated. Laterality was determined via a behaviorally validated 13-item questionnaire which assesses four types of lateral preference (hand, foot, eye, and ear; Coren, Porac, \& Duncan, 1979). Self-report items are scored as +1 for right-, -1 for left-, and 0 for either-hand dominance. Criterion for right dominance and inclusion in the experiment was a score of +8 or above ( $\max =$ $+13)$. Mean laterality score was $11.84(S D=1.59)$. The subjects were screened to exclude for known neurological or visual impairments. Corrected-to-normal vision was not a basis for exclusion.

\section{Tachistoscopic Apparatus and Materials}

Eighteen faces - nine happy and nine angry - were randomly selected from Ekman and Friesen's (1978) pictures of facial affect. Slides of these pictures were prepared with the stimulus face appearing in either the RVF or the LVF and were mounted with the inside edge of the picture $3^{\circ}$ from center and the outside edge $12^{\circ}$ from center (see Sergent, 1982). A total of 36 slides ( 9 RVF happy, 9 LVF happy, 9 RVF angry, and 9 LVF angry) were created.

The experimental chamber was sound attenuated, and the automated programming equipment and experimenter were located in a separate room. The subjects were monitored through a one-way observation window and prompted via an intercom. A constantillumination tachistoscope (Lafayette Instruments 42011) presented the stimuli onto a screen $2.67 \mathrm{~m}$ in front of the subject. The center of the screen was marked with a black dot positioned $1.47 \mathrm{~m}$ above the floor and at the subject's eye level. Tachistoscopic-trial onset was signaled by a $2000 \mathrm{~Hz}, 55 \mathrm{~dB}$ (A-scale) tone located behind the subject. The manipulanda consisted of two "soft touch" trip switches flush-mounted midline on a right-handed student desk $58.5 \mathrm{~cm}$ from the back of the chair. Response keys, separately labeled "Happy" and "Angry," were counterbalanced across subjects to eliminate position effects.

\section{Experimental Conditions and Materials}

The experiment consisted of two priming conditions - affective subvocal rehearsal and neutral subvocal rehearsal-and a control condition. The control condition assessed baseline performance in each visual field. Subvocal rehearsal of three neutral words (e.g., chance, law, method) and three affective words (e.g., hate, kill, rape) was required in the neutral and affective subvocal rehearsal conditions, respectively. On the basis of the valence hypothesis, which indicates that the right-hemisphere processes negative affect (Borod, 1992), only negative affective words were used in the affective-word condition. Words in both conditions were one- or two-syllable words matched on frequency of occurrence (Thorndike \& Lorge, 1944) and number of syllables. No subvocal rehearsal was required in the control condition.

These three conditions constituted an experimental trial. The experiment was composed of four trials, each separated by a 1-min interval. Conditions were counterbalanced within the experimental trials. 


\section{Tachistoscope Presentations}

Six slides were tachistoscopically presented in each experimental condition. This allowed three slides of each affective valence-angry and happy-to be presented in each condition. The slides were pseudorandomized into six orders, with the provision that no more than two consecutive slides should have the same affect or visual field. Since three conditions constituted an experimental trial, 18 slides were presented in each trial, with a total of 72 slides being presented across the four trials of the experiment. This permitted six slides per permutation of visual field, affect, and condition.

\section{Procedures}

The seated subjects completed the informed consent form and the handedness questionnaire. Tape-recorded tachistoscope instructions for the control condition were then presented. The subjects were told that each slide would be preceded by a 1-sec tone and that, upon hearing it, they were to fixate on the black dot because the slide would be presented $3 \mathrm{sec}$ after the tone. They were instructed to decide as quickly as possible whether the presented face was happy or angry. They then completed a practice trial consisting of 10 happy and 10 angry slides. A second set of taperecorded instructions, this time for the priming tasks, informed the subjects to neither imagine nor picture the words. Another practice trial was then presented. The subjects were required to achieve an accuracy level of at least $80 \%$ on both practice trials for inclusion in the experiment.

In each priming condition, the experimenter orally presented three words to the subject via the intercom. Following each list of words, whether affective or neutral, the command "Rehearsal" notified the subjects to begin rehearsal. After they had subvocally rehearsed the words for five sec, a 1-sec tone sounded, signaling the beginning of the six tachistoscopic presentations and the presentation of the first slide. The same $1-\mathrm{sec}$ tone signaled the presentation of each slide, and $3 \mathrm{sec}$ later, the slide was shown for $200 \mathrm{msec}$. This exposure duration was used to reduce errors (see Sergent, 1982). Each slide presentation was separated by $10 \mathrm{sec}$. Following completion of the condition, a tone notified subjects to

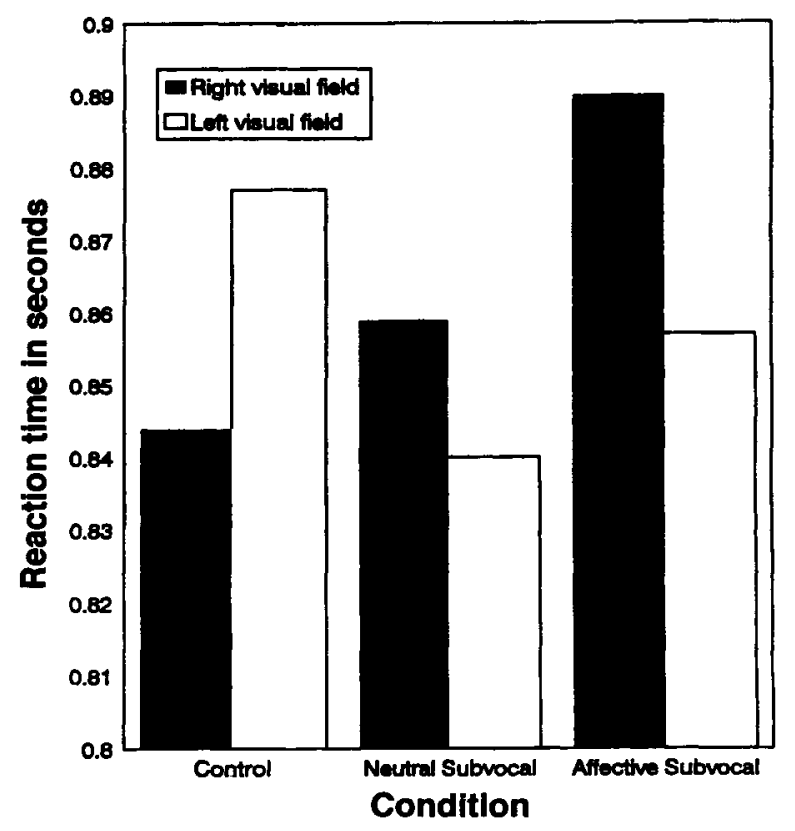

Figure 1. Mean reaction time in sec as a function of condition and visual field.

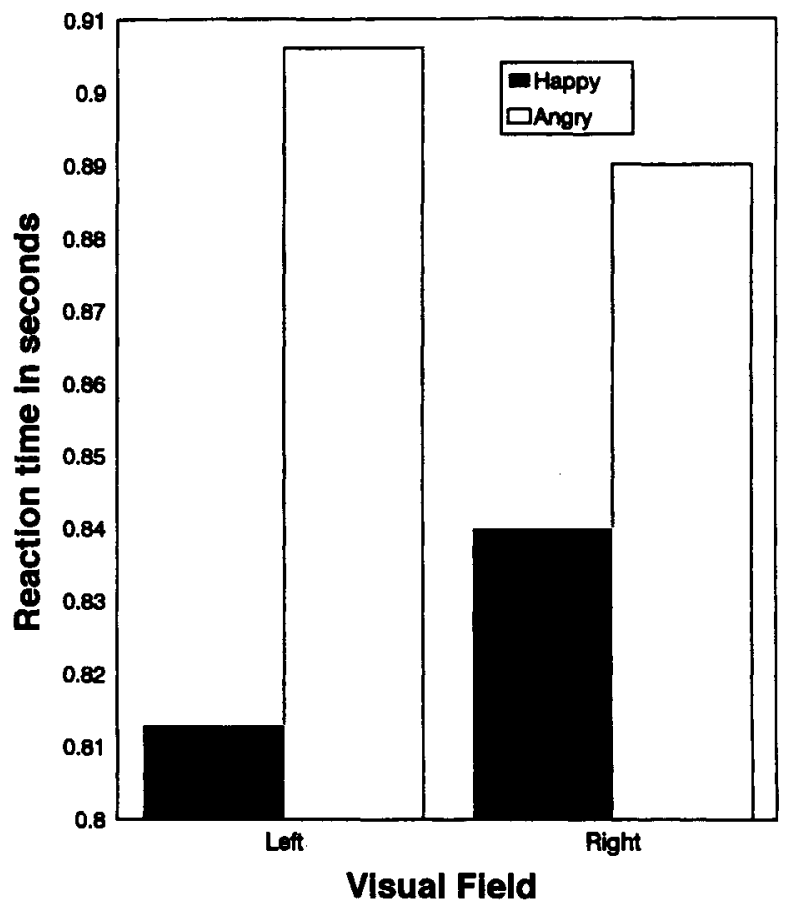

Figure 2. Mean reaction time in sec as a function of visual field and affect.

verbally recall the presented words. Recall percentage was computed to ensure compliance with the task. No words were presented in the control condition, and the subjects sat quietly for five sec prior to the tachistoscopic presentations. Each experimental condition was separated by $20 \mathrm{sec}$. The subjects were reminded to focus on the fixation point after each trial to improve integrity of the stimulus presentation within each visual field.

\section{RESULTS}

Reaction-time means for correct responses were analyzed with a three-factor fully repeated measures analysis of variance (ANOVA), with factors of visual field (left, right), affect (happy, angry), and condition (control, neutral subvocal rehearsal, affective subvocal rehearsal). Conservative degrees of freedom were used to determine the significance level of the interactions, and only the adjusted degrees of freedom are reported (Greenhouse \& Geisser, 1959; Hays, 1988). Pairwise comparisons of means were performed using Duncan's Multiple Range Test (alpha $=.05$ )

Errors $(N=106)$ occurred on $4.7 \%$ of the slide presentations. Responses greater than $2.0 \mathrm{sec}$ were considered outliers $(N=15)$ and were not included in the data analyses. Chi-squares indicated that there was a nonsignificant trend for more errors to be made on angry than on happy slides $\left[\chi^{2}(1)=3.6, p<.10\right]$, but that there were no differences in error frequency across the visual fields $\left[\chi^{2}(1)=2.38\right.$, n.s. $]$ or across conditions $\left[\chi^{2}(2)=.46\right.$, n.s. $]$.

The ANOVA on reaction-time data revealed a significant condition $\times$ visual field interaction $[F(1,30)=$ $8.84, p<.007$; see Figure 1]. Post hoc comparisons in- 
dicate a significant RVF advantage in the control condition but a reversal in favor of the LVF in the affective rehearsal condition. A similar, though nonsignificant, pattern was apparent in the neutral rehearsal condition. RVF performance was slower in both rehearsal conditions relative to the control condition, but only significantly so in the affective rehearsal condition. Conversely, LVF reaction time improved in both rehearsal conditions relative to the control condition. Overall, subvocal rehearsal of affective and neutral words interfered with RVF performance, but facilitated LVF performance.

Manipulation checks revealed the ease of both subvocal rehearsal tasks. Word-recall accuracy following the neutral and affective subvocal rehearsal conditions was $100 \%$ and $99.7 \%$, respectively.

The visual field $X$ affect interaction was also significant $[F(1,30)=4.66, p<.039$; see Figure 2]. Post hoc comparisons indicate that angry faces were perceived equally across visual fields, but that there was an LVF advantage for happy faces. The main effect of affect was highly significant $[F(1,30)=23.34, p<.0001]$, with reduced reaction time for happy faces.

\section{DISCUSSION}

There are two main findings of this experiment. First, contrary to our hypotheses, subvocalization of both affective and neutral words interfered with RVF performance and facilitated LVF performance. Second, angry faces were perceived equally across visual fields, but an LVF advantage was observed for happy faces. Implications of these findings are discussed below.

Initially conceptualized to explain priming effects, the selective activation model was later refined to also account for interference, which results when two tasks compete for similar or adjacent cerebral areas within the same hemisphere under heavy load conditions (Kinsbourne, 1980). Tasks represented in adjacent regions are thought to be affected via spreading activation (i.e., neural activity) in a tightly connected cerebral network (Kinsbourne \& Byrd, 1985). The interference of RVF performance during subvocalization thus implicates an overloading of the affective perceptual capacities of the left hemisphere by the linguistic requirements of word rehearsal. This effect was observed for rehearsal of both neutral and affective words, suggesting that, contrary to our hypothesis, all verbal material is represented in, and processed by, the left hemisphere.

These interference effects are inconsistent with recent dual-task research which has demonstrated priming effects with similar simple and noneffortful tasks (Kinsbourne \& Byrd, 1985; Van Strien \& Bouma, 1990). Though manipulation checks indicate the apparent ease of these tasks, they may not have been sensitive enough to evaluate the tasks' effort requirements. Specifically, word-recall percentage may poorly reflect the effort or processing demands of verbal rehearsal during the six slide presentations. In contrast, Van Strien and Bouma (1990) found priming effects when subvocal rehearsal was required during only one slide presentation. Despite the manipulation checks, the length of the experimental tasks suggest that they required more effort and processing than initially hypothesized. Fewer trials than the six presentations per condition would decrease the load requirement and potentially allow emergence of lefthemisphere priming effects.

In addition to RVF interference, robust LVF performance improvements are apparent which are poorly accounted for by Kinsbourne's model. The pattern of results is striking, showing an RVF advantage in the control condition, but a shift in favor of the LVF in the rehearsal conditions. This reflects a shift in the relative balance of activation between the hemispheres in favor of the right hemisphere. Similar hemispheric shifts have been observed under stressful conditions (Gruzelier \& Phelan, 1991) and with the threat of electric shock (Asbjørnsen, Hugdahl, \& Bryden, 1992). Gruzelier and Phelan (1991) found a reversal of an initial RVF advantage in a lexical visual-field task in medical students prior to an exam, whereas Asbjørnsen et al. (1992) demonstrated that threat of electric shock abolished the right-ear advantage in a dichotic listening lexical task by increasing left-ear performance and by decreasing rightear performance. Asbjørnsen et al. (1992) posited that the heightened arousal associated with the potential shock preferentially activated the right hemisphere and facilitated callosal transfer of the left-ear signal. Reduction of right-ear performance was explained in terms of the left-ear signal interfering with the same resources that activated the right-ear signal. In brief, findings of preferential right-hemisphere involvement under stressand arousal-inducing conditions are consistent with theorizing which submits that the right hemisphere is preferentially involved in bilateral cerebral activation and arousal (Heilman \& Van Den Abell, 1979; Tucker, Roth, Arneson, \& Buckingham, 1977).

The callosal-transfer notion presented above may account for the pattern of results obtained in this experiment. The heavy processing requirements of the rehearsal tasks were sufficiently arousal-inducing to preferentially prime or activate the right hemisphere. Callosal transfer of LVF material was also facilitated, which improved right-hemisphere processing but interfered with RVF material and left-hemisphere processing. The balance of activation between the hemispheres therefore shifted from a significant RVF advantage (control condition) to a trend toward an LVF advantage (neutral rehearsal), ending up with a significant LVF advantage (affective rehearsal). Hence, the affective rehearsal condition, with its negative affective words, was most arousal inducing, robustly improving LVF performance but compromising RVF performance. Overall, this approach accounts for the LVF improvements by positing righthemisphere priming; in contrast to Kinsbourne's model, however, it highlights callosal inhibition of RVF mater- 
ial to explain the left-hemisphere decrement. Whether RVF interference is a function of this inhibition, an overloading of the left hemisphere's perceptual capacities, or some combination of the two cannot be determined with this paradigm.

Further, in the present experiment, a right-hemisphere advantage for processing happy faces was obtained. A substantial corpus of research has examined hemispheric asymmetries in affect perception, and the findings have diverged considerably. There are data, for instance, that support a right-hemisphere advantage for both positively and negatively valenced affective faces (Buchtel, Campari, De Risio, \& Rota, 1978; Ley \& Bryden, 1979; Strauss \& Moscovitch, 1981), those that support a right-hemisphere bias for sad faces and a lefthemisphere bias for happy faces (Reuter-Lorenz \& Davidson, 1981), and those that support a righthemisphere advantage for only happy faces (Duda \& Brown, 1984; Hugdahl, Iversen, Ness, \& Flaten, 1989). Despite disparate methodologies, our findings are consistent with Duda and Brown's (1984) interaction (i.e., visual field $X$ affect) and main effect for happy faces. They submit that the happy faces in Ekman's standardized faces may be more discriminable than either neutral or negative faces. If so, the more pronounced facial features of happy faces may be better suited for the putative holistic processing of the right hemisphere (see Sergent, 1982). Nonetheless, our affective asymmetry findings should be interpreted cautiously, since they were obtained in a dual-task paradigm whose methodology differs substantially from that of many tachistoscope experiments.

Overall, subvocal rehearsal of neutral and affective words interfered with RVF performance, but facilitated that of the LVF. Right-hemisphere priming may be considered a function of the arousal-inducing character of the rehearsal tasks, and left-hemisphere interference may be viewed as the result of both inhibition of callosal transfer and an overloading of its perceptual capacities. The precise contribution of the latter two processes in left-hemisphere interference remains unknown. Future dual-task research should vary the processing requirements of similar manipulations, including the number of words and length of rehearsal. Tasks with less effort should improve performance in the relevant visual field, though, as demonstrated in this experiment, tasks with substantial effort and processing requirements are hypothesized to preferentially activate the right hemisphere.

\section{REFERENCES}

AsbJørnsen, A., Hugdahl, K., \& Bryden, M. P. (1992). Manipulations of subjects' level of arousal in dichotic listening. Brain $\&$ Cognition, 19, 183-194.

BOLES, D. B. (1979). Laterally biased attention with concurrent verbal load: Multiple failures to replicate. Neuropsychologia, 17, 353-361.

Boller, F., Cole, M., Vrtunski, P. B., Patterson, M., \& Kim, Y. (1979). Paralinguistic aspects of auditory comprehension in aphasia. Brain \& Language, 7, 164-174.
BOROD, J. C. (1992). Interhemispheric and intrahemispheric control of emotion: A focus on unilateral brain damage. Journal of Consulting \& Clinical Psychology, 60, 339-348.

Borod, J. C., Andelman, F., Obler, L. K., Tweedy, J. R., \& WelkowITZ, J. (1992). Right hemisphere specialization for the identification of emotional words and sentences: Evidence from stroke patients. Neuropsychologia, 30, 827-844.

Bouma, A. (1987). Effects of concurrent spatial and verbal memory loads on serial position functions of laterally presented letter strings. Brain \& Cognition, 6, 295-320.

Brody, N., Goodman, S., Holm, E., Krinzman, S., \& Sebrechts, M. (1987). Lateralized affective priming of lateralized affectively valued target words. Neuropsychologia, 25, 935-946.

BRYDEN, M. P., \& LEY, R. G. (1983). Right hemisphere in imagery and affect. In E. Perecman (Ed.), Cognitive processing in the right hemisphere. New York: Academic Press.

Buchtel, H., Campari, F., De Risio, C., \& Rota, R. (1978). Hemispheric differences in discriminative reaction time to facial expression. Italian Journal of Psychology, 5, 159-169.

Coren, S. P., Porac, C., \& DunCan, P. (1979). A behaviorally validated self-report inventory to assess 4 types of lateral preferences. Journal of Clinical Neuropsychology, 1, 55-64.

Davidson, R. J. (1992). Anterior cerebral asymmetry and the nature of emotion. Brain \& Cognition, 20, 125-151.

Davidson, R. J., Ekman, P., Saron, C. D., Senulis, J. A., \& Friesen, W. J. (1990). Approach-withdrawal and cerebral asymmetry: Emotional expression and brain physiology I. Journal of Personality \& Social Psychology, 58, 330-341.

Demakis, G. J., Harrison, D. W., \& Campen, M. J. (1993). A test of Kinsbourne's selective activation model. International Journal of Neuroscience, 72, 201-207.

DuDA, P. O., \& BROWN, J. (1984). Lateral asymmetry of positive and negative emotions. Cortex, 20, 253-261.

Ekman, P., \& Friesen, W. (1978). Pictures of facial affect. Palo Alto, CA: Consulting Psychologists Press.

GAINOTTI, G. (1972). Emotional behavior and hemispheric side of lesion. Cortex, 8, 41-55.

GARDNER, E. B., \& BRANSKI, D. M. (1976). Unilateral cerebral activation and perception of gaps: A signal detection analysis. Neuropsychologia, 14, 43-53.

GreEnhouse, S. W., \& Geisser, S. (1959). On methods in the analysis of profile data. Psychometrika, 24, 95-112.

Gruzelier, J., \& Phelan, M. (1991). Stress induced reversal of a lexical divided visual-field asymmetry accompanied by a retarded electrodermal habituation. International Journal of Psychophysiology, 11, 269-276.

Harrison, D. W., \& Gorelczenko, P. (1990). Functional asymmetry for facial affect perception in high and low hostile men and women. International Journal of Neuroscience, 53, 1-9.

HAYS, W. L. (1988). Statistics. Chicago: Holt, Rinehart \& Winston.

Heilman, K. M., \& VAN Den Abell, T. (1979). Right hemisphere dominance for mediating cerebral activation. Neuropsychologia, 17, 315-321.

Hellige, J. B., \& Cox, P. J. (1976). Effects of concurrent verbal memory on recognition of verbal stimuli from the left and right visual field. Journal of Experimental Psychology: Human Perception \& Performance, 2, 210-221.

Hellige, J. B., Cox, P. J., \& LitvaC, L. (1979). Information processing in the cerebral hemispheres: Selective hemispheric activation and capacity limitation. Journal of Experimental Psychology: General, 108, 251-279.

Hugdahl, K, Iversen, P. M., Ness, H., \& Flaten, M. A. (1989). Hemispheric differences in recognition of facial expressions: A VHF study of negative, positive, and neutral emotions. International Journal of Neuroscience, 45, 205-213.

Kinsbourne, M. (1970). The cerebral basis of lateral asymmetries in attention. Acta Psychologica, 33, 193-201.

KINSBOURNE, M. (1980). A model for the ontogeny of cerebral organization in non-right handers. In J. Herron (Ed.), Neuropsychology of left-handedness. New York: Academic Press.

Kinsbourne, M., \& Byrd, M. (1985). Word list and visual hemifield 
shape recognition: Priming and interference effects. In M. J. Posner \& O. S. M. Marin (Eds.), Mechanisms of attention: Attention and performance IX. Hillsdale, $\mathrm{NJ}$ : Erlbaum.

Kinsbourne, M., \& Hisock, M. (1983). Asymmetries of dual-task performance. In J. B. Hellige (Ed.), Cerebral hemisphere asymmetries: Method, theory and application. New York: Praeger.

Landis, T., Graves, R., \& Goodglass, H. (1982). Aphasic reading and writing: Possible evidence for right hemisphere participation. Cortex, 18, 105-112.

LEY, R. G., \& BRYDEN, M. P. (1979). Hemispheric differences in processing emotions and faces. Brain \& Language, 7, 127-138.

Metzger, R. L., \& ANTES, J. R. (1976). Sex and coding strategy effects on reaction time to hemispheric probes. Memory \& Cognition, 4, 167-171.

Moscovitch, M. (1986). Afferent and efferent models of visual perceptual asymmetries: Theoretical and empirical implications. Neuropsychologia, 24, 91-114.

REUTER-LORENZ, P., \& DAVIDSON, R. J. (1981). Differential contributions of the two cerebral hemispheres to the perception of happy and sad faces. Neuropsychologia, 19, 609-613.

Seamon, J. C., \& Gazzaniga, M. S. (1973). Coding strategies and cerebral laterality effects. Cognitive Psychology, 5, 249-256.
SERGENT, J. (1982). Theoretical and methodological consequences of variations in exposure duration in visual laterality studies. Perception \& Psychophysics, 31, 451-461.

Strauss, E., \& Moscovitch, M. (1981). Perception of facial expressions. Brain \& Language, 13, 308-322.

THORNDIKE, E. L., \& LoRGE, I. (1944). The teachers' word book of 30,000 words. New York: Teachers College Press, Columbia University.

Tucker, D. M., Roth, R. S., Arneson, B. A., \& Buckingham, V. (1977). Right hemisphere activation during stress. Neuropsychologia, 15, 697-700.

VAN Strien, J. W., \& Bouma, A. (1990). Selective activation effects of concurrent verbal and spatial memory loads in left-handed and right-handed adults. Brain \& Cognition, 14, 81-91.

VAN Strien, J. W., \& MorPuRgo, M. (1992). Opposite hemispheric activations as a result of emotionally threatening and non-threatening words. Neuropsychologia, 38, 845-848.

(Manuscript received December 21, 1993; revision accepted for publication May 26, 1994.) 